\title{
Cross-talk between microtubules and the linker of nucleoskeleton complex plays a critical role in the adipogenesis of human adipose-derived stem cells
}

Yiting Yang ${ }^{1 \dagger}$, Rongmei $\mathrm{Qu}^{1 \dagger}$, Tingyu Fan ${ }^{1}$, Xi Zhu ${ }^{2}$, Yanting Feng ${ }^{1}$, Yuchao Yang ${ }^{1}$, Ting Deng ${ }^{1}$, Yan Peng ${ }^{1}$, Wenhua Huang ${ }^{1 *}$, Jun Ouyang ${ }^{1 *}$ and Jingxing Dai ${ }^{1 *}$ (D)

\begin{abstract}
Background: Adipose-derived stem cells (ASCs) that show multidifferentiation and anti-immune rejection capacities have been widely used in plastic and reconstructive surgery. Previous studies have indicated that mechanical and biophysical interactions between cells and their surrounding environment regulate essential processes, such as growth, survival, and differentiation, and the cytoskeleton system plays an important role in the mechanotransduction. However, the role of mechanical force in the determination of lineage fate is still unclear.

Methods: Human ASCs (hASCs) were obtained from three different donors by liposuction. Adipogenesis and osteogenesis were determined by Oil Red $O$ and Alizarin Red staining, respectively. The mRNA levels of the cytoskeleton system, PPARY, and C/EBPa were determined by real-time polymerase chain reaction (RT-PCR). The level of cytoskeleton, PPARY, and C/EBPa protein levels were measured by Western blotting. The morphology of the cytoskeleton system during adipogenesis was observed with confocal microscopy. hASCs were transfected with a SUN2-specific shRNA to knockdown sun2, and a nontargeting shRNA was used as a control.

Results: We found that disrupting the physiological balance between the cytoskeleton and the linker of the nucleoskeleton and cytoskeleton (LINC) complex (especially SUN2) could impact the adipogenesis of hASCs in vitro. Microtubule (MT) depolymerization with nocodazole (which interferes with the polymerization of MTs) increased the expression of SUN2 and PPARY, while taxol (an inhibitor of MT disassembly) showed the opposite results. Meanwhile, hASCs with sun2 knockdown overexpressed MTs and decreased PPARY expression, thereby inhibiting the adipogenesis. Furthermore, knockdown of sun2 changed the structure of perinuclear MTs.

Conclusions: We demonstrated the presence of cross-talk between MT and SUN2, and this cross-talk plays a critical role in the rebalance of the mechanical environment and is involved in the regulation of PPARY transport during adipogenic differentiation of hASCs.
\end{abstract}

Keywords: Human adipose-derived stem cells (hASCs), Sad1/UNC-84 2 (SUN2), The linker of the nucleoskeleton and cytoskeleton (LINC) complex, Microtubules (MTs), Mechanotransduction

\footnotetext{
* Correspondence: 13822232749@139.com; jouyang@126.com;

daijx2013@163.com

†Equal contributors

${ }^{1}$ Department of Anatomy, Guangdong Provincial Key Laboratory of Medicine

and Biomechanics, School of Basic Medical Sciences, Southern Medical

University, Guangzhou 510515, China

Full list of author information is available at the end of the article
} 


\section{Background}

Autologous fat transfers have been successfully used for structural fat grafting in facial, lip, and hand rejuvenation and body contour improvement. One of the most interesting areas of recent research is adipose-derived stem cells (ASCs) and their potential for tissue remodeling and differentiation into specialized somatic cell types to replace damaged tissues and organs, as well as their use in cosmetic, plastic, and reconstructive surgery $[1,2]$. Multiple factors determine stem cell fate, including chemical, temporal, spatial, and physical clues. However, although the regulation of stem cell differentiation by soluble factors is well characterized, the role of mechanical force in the determination of lineage fate is still unclear.

Physical cues play an important role in the determination of lineage fate in stem cells. Mechanical and biophysical interactions between cells and their surrounding environment regulate essential processes, such as growth, survival [3], and differentiation [4]. Integrin-mediated adhesion and interaction with cytoskeleton components (especially actin) are classic physical connections between cells and the extracellular matrix that regulate various aspects of cellular mechanotransduction [5]. Recent studies have suggested that the nucleus and the nuclear envelope (NE) act as mechanosensory organelles, where anchoring to the cytoskeleton through the linker of the nucleoskeleton and cytoskeleton (LINC) complex enables the transmission of mechanical force between the nucleus and the cytoskeleton outward to the external microenvironment $[6,7]$. Previous studies have shown that SUN1 and SUN2 interact with lamins, other inner nuclear membrane proteins, Nesprin-1, -2 , and -3 , associate with actin, microtubules (MTs), and/or intermediate filaments in the cytoplasm [8]. For instance, Nesprin-1 and -2 (SYNE1, SYNE2) exist in numerous splice isoforms. The giant isoforms of Nesprin-1 and Nesprin-2 interact with actin via their $\mathrm{N}$-terminal actin-binding domain, whereas other isoforms can associate with microtubule motors $[9,10]$.

Genetic elements of the nucleus have been shown to respond to mechanical challenges indirectly through their transduction into intermediary biochemical cascades [11], and a hypothesis has been proposed whereby applied forces might directly alter chromosomal conformation and influence the accessibility of genetic information during the binding of transcriptional enhancers or repressors [12]. However, the details of the biophysical mechanisms that control this sensitivity remain unclear.

In the process of adipogenic differentiation, human ASCs (hASCs) are transformed into lipid-filled morphologically spherical mature adipocytes. This change suggests that the transition from adult stem cells to mature adipocytes involves significant cytoskeletal reorganization or cytoskeleton remodeling [13]. Indeed, the disruption of F-actin stress fibers, which are major components of the cytoskeleton, leads to increased intracellular triacylglycerol accumulation and significantly enhanced adipogenesis [14]. Other components of the cytoskeleton have also been linked with adipogenesis, such as vimentin, which forms a cage-like structure surrounding initial lipid droplets (LDs) [15]. These studies suggest that cytoskeleton remodeling and force rebalancing is a limiting step in the morphological transition during adipogenic differentiation. However, it is not clear how remodeling of the LINC complex is regulated and initiated during adipogenesis. In this study, we investigated the changes in microtubules and the LINC complex, and we also examined its regulation by SUN2 during adipogenesis in hASCs to demonstrate the interaction between microtubules and the LINC complex and the possible mechanisms of adipogenic differentiation of ASCs.

\section{Methods \\ Cell culture}

hASCs were characterized for surface markers (Additional file 1: Figure S1). For adipogenic and osteogenic differentiation, Oil Red $\mathrm{O}$ and Alizarin Red staining were performed as described previously [16, 17]. hASCs between three and six passages were used in the experiments, and all experiments were repeated at least three times using hASCs from three donors. hASCs were cultured in growth medium consisting of high-glucose Dulbecco's modified Eagle's medium (DMEM; Gibco), 10\% fetal bovine serum (FBS; Gibco), and 1\% penicillin/streptomycin (Gibco).

For the adipogenesis experiments, postconfluent hASCs were cultured with adipogenic induction medium (AIM; $10 \% \mathrm{FBS}, 1 \%$ penicillin/streptomycin, $10 \mu \mathrm{g} / \mathrm{ml}$ insulin (Sigma), $10 \mu \mathrm{M}$ indomethacin (Sigma), $100 \mathrm{nM}$ dexamethasone (Sigma), and $0.5 \mathrm{mM}$ 3-isobutyl methylxanthine (Sigma)) for 3 days. The cells were then cultured with maintenance medium (AMM; 10\% FBS, 1\% penicillin/streptomycin, $10 \mu \mathrm{g} / \mathrm{ml}$ insulin, $10 \mu \mathrm{M}$ indomethacin, $100 \mathrm{nM}$ dexamethasone) for 1 day. The cells were then cultured with AIM to the time point for further experiments. Taxol (Selleck) was used at $10 \mathrm{nM}$ and nocodazole (Sigma) was used at $0.1 \mu \mathrm{g} / \mathrm{ml}$, except where noted in the figure legends. Taxol and nocodazole were added when AIM was used the first time. The cells were then cultured with AMM for 1 day. After that, the cells were cultured with AIM (without taxol and nocodazole) to the time point for further experiments (Additional file 2: Figure S2). All cells were maintained at $37{ }^{\circ} \mathrm{C}$ in a $5 \% \mathrm{CO}_{2}$ humidified incubator.

For the osteogenesis experiments, we cultured ASCs with osteogenic induction medium (10\% FBS, 1\% penicillin/ streptomycin, $100 \mathrm{nM}$ dexamethasone, $200 \mu \mathrm{M}$ ascorbic acid (Sigma), and $10 \mathrm{mM}$ glycerol 2-phosphate (Sigma)). 


\section{Lentivirus-mediated transduction}

For knockdown of human SUN2, a SUN2-specific short hairpin RNA (shRNA: 5'-CCGGCTGCAGAAA GAAGGTGTGATTCTCGAGAATCACACCTTCTTTCT GCAGTTTTTG -3') was packaged into a lentivirus from Genechem (Shanghai, China) (Additional file 3: Figure S4). For infection of target cells, the viral supernatants were diluted fourfold in fresh medium before transduction. Transduction was carried out in the presence of $5 \mu \mathrm{g} / \mathrm{ml}$ polybrene, and the medium was changed $12 \mathrm{~h}$ later. For the establishment of stable clones, $2 \mu \mathrm{g} / \mathrm{ml}$ puromycin (Santa Cruz) was added to the culture medium, and the fresh puromycin-containing medium was replaced every 3-4 days until resistant colonies were identified.

\section{Oil Red $O$ staining}

For Oil Red O staining, cells cultured on a cover glass were rinsed with phosphate-buffered saline (PBS), fixed with $4 \%$ paraformaldehyde (Tiengen Biological) for 10 min, and washed with 60\% isopropyl alcohol (Tiengen Biological). Cells were then incubated in 2\% $(w / v)$ Oil Red $\mathrm{O}$ reagent (Cyagen) for $1 \mathrm{~h}$ at room temperature. Excess staining was removed by washing with $60 \%$ isopropyl alcohol, followed by several washes with distilled water. The percentage of adipogenic differentiation was determined by counting Oil Red O-positive and -negative cells (every 100 cells/field in five fields) by microscopy.

\section{Immunofluorescence staining}

hASCs cultured on glass coverslips were fixed in $4 \%$ paraformaldehyde in PBS for $10 \mathrm{~min}$ at room temperature and washed with PBS. Cells were then permeabilized in $0.1 \%$ Triton X-100 in PBS at room temperature for $10 \mathrm{~min}$, blocked using blocking buffer (15\% goat serum in PBS) for $1 \mathrm{~h}$, and subsequently incubated with primary antibodies diluted in blocking buffer overnight. In addition to the primary antibodies in the previous paragraph, antibodies against $\alpha$ tubulin (1:800; mouse; clone DM1A; Abcam), lamin A (1:400; mouse; clone 133A2; Abcam), SUN2 (1:400; rabbit; Abcam), $\beta$-actin (1:500; rabbit; Sigma-Aldrich), Nesprin-1 (1:400; rabbit; clone EPR14196; Abcam), and Nesprin-3 (1:400; rabbit; Abcam) were used for immunostaining cells. Coverslips were washed with PBS for 15 min and incubated with Alexa Fluor 488conjugated (1:500; ThermoFisher), Alexa Fluor 568conjugated (1:500; ThermoFisher), and Alexa Fluor 633-conjugated (1:500; ThermoFisher) secondary antibodies diluted in blocking buffer at room temperature for $1 \mathrm{~h}$. Coverslips were washed and mounted on to slides in 4',6-diamidino-2-phenylindole (DAPI) mounting medium (Santa Cruz Biotechnology) and then imaged with confocal microscopy (Zeiss).

\section{Western blotting analysis}

Cells were collected in a pH 8.0 lysis buffer $(50 \mathrm{mM}$ Tris$\mathrm{HCl}, 120 \mathrm{mM} \mathrm{NaCl}$, and 0.5\% NP-40). Protein concentrations in the extracts were estimated using the Bradford method. A total of $20 \mu \mathrm{g}$ of protein sample was loaded per lane, separated by SDS-PAGE, and then transferred to PVDF membranes. Membranes were subsequently blocked in 10\% nonfat milk (Difco ${ }^{\text {ma }}$ Skim Milk; BD Biosciences) in TBS with Tween 20 (TBST) for $1 \mathrm{~h}$ at room temperature and incubated with glyceraldehyde-3-phosphate dehydrogenase (GAPDH; 1:1000; rabbit; Cell Signaling), SUN1 (1: 1000; clone EPR6554; rabbit; Abcam), SUN2 (1:1000; clone EPR6557; rabbit; Abcam), $\beta$-actin (1:500; rabbit; SigmaAldrich), $\alpha$-tubulin (1:1000; clone DM1A; Abcam), lamin A (1:500; mouse; clone 133A2; Abcam), Nesprin-1 (1:400; clone EPR14196; rabbit; Abcam), Nesprin-2 (1:400; mouse; Abcam), Nesprin-3 (1:400), PPAR (1:1500; rabbit; clone K. 242.9; ThermoFisher), and KIF5B (1:800; rabbit; Abcam) primary antibodies at $4{ }^{\circ} \mathrm{C}$ overnight. The membranes were washed for $15 \mathrm{~min}$ in TBST and then incubated for $1 \mathrm{~h}$ with horseradish peroxidase (HRP)-conjugated secondary antibodies (1:5000; Pierce) in TBST at room temperature. After washes with TBST, the membranes were visualized by enhanced chemiluminescence (ECL) detection reagents (Applygen, China). Immunoreactive bands were detected by the ECL detection system (Protein Simple, USA), and densitometric values were analyzed with Quantity One v4. 6.2 software (VersaDoc; Bio-Rad). The relative expression of each immunoreactive band was normalized to GAPDH.

\section{RNA extraction and quantitative real-time polymerase chain reaction analysis}

Total RNA from hASCs was extracted using TRIzol reagent (Invitrogen, USA) according to instructions provided by the manufacturer. Total RNA $(1 \mu \mathrm{g})$ was then used for reverse transcription (RT) with a commercially available kit (Revert Aid First Strand cDNA Synthesis Kit, Fermentas). Real-time polymerase chain reaction (PCR) was performed in triplicate with an ABI Step One Plus system (Applied Biosystems, USA) and a fluorescencelabeled SYBR Green/ROX qPCR Master Mix kit (Fermentas) using specific primers for $\alpha$-tubulin, $\beta$-actin, SUN2, lamin A, Nesprin-2, Nesprin-3, C/EBP $\alpha$, PPAR $\gamma$, and GAPDH, which was used as an endogenous control. For quantification, the point of product accumulation in the early logarithmic phase of the amplification plot was defined by assigning a fluorescence threshold above the background, defined as the threshold cycle $(\mathrm{Ct})$ number. Relative expression of different gene transcripts was calculated by the $\Delta \Delta \mathrm{Ct}$ method. The Ct of any gene of interest was normalized to the $\mathrm{Ct}$ of the normalizer (GAPDH). Fold changes (arbitrary units) were determined as $2-\Delta \Delta \mathrm{Ct}$. 


\section{Image analysis}

Following immunofluorescence experiments, raw image data were analyzed using Image ${ }^{6}$ to quantify the nuclear deformation. Simultaneously, each selection was fitted to an ellipse from which we obtained the lengths of the major and minor axes and the size of the lipid droplets. Additionally, the fluorescence intensities of Nesprin-1, Nesprin-3, and $\alpha$-tubulin were measured with the Image ${ }^{6}$ software.

\section{Statistical analysis}

At least three independent experiments were carried out unless otherwise stated. The results are reported as the mean \pm standard deviation and were analyzed using unpaired Student's t test. The significant difference levels were set at ${ }^{*} P<0.05$, ${ }^{* *} P<0.01$, and ${ }^{* * *} P<0.001$.

\section{Results}

Microtubule-based cytoskeleton reconstruction during adipogenesis

First, we analyzed cell morphology and the expression of $\alpha$-tubulin at the protein level during adipogenesis of hASCs at various time points. Adipogenic differentiation was evaluated by the adipogenic markers PPARY and $\mathrm{C} /$ EBP $\alpha$ after 14 days. In undifferentiated hASCs (day 0), microtubules were well organized in a regular array. When cells were undergoing differentiation, after the initiation of adipogenesis (day 4), the microtubule density increased around the nucleus, while peripheral microtubules became sparse and were arranged into a vacuolar structure. At the middle stage of adipocyte differentiation (day 7), microtubules remained around the nucleus at a high density and formed a cobweb-like structure. Peripheral microtubules in vacuoles fused together and gradually formed a larger gap. As cells further matured (day 14), the microtubule density around the nucleus was reduced, and microtubules translocated to the peripheral cytoplasm (Fig. 1a, c).

To detect the variations in microtubules during adipogenesis, we measured the mRNA and protein levels of $\alpha$-tubulin by qRT-PCR and Western blotting analyses at various time points (days 0, 4, 7, and 14) during adipogenic differentiation. The mRNA level and also the protein level of $\alpha$-tubulin was decreased during adipogenesis (Fig. 1d, e).

To determine the effects of changes in the status of microtubule polymerization on adipogenesis, we treated hASCs with nocodazole, which interferes with microtubule polymerization and promotes microtubule disassembly, or with Taxol, which stabilizes microtubules [18]. Fourteen days after treatment with the adipogenic cocktail, microtubule density had decreased in nocodazoletreated cells, while microtubules had accumulated in the nucleus in taxol-treated cells (Fig. 2i). The total lipid content and the size of the lipid droplets increased in nocodazole-treated cells but decreased in taxol-treated cells (Fig. $2 \mathrm{a}-\mathrm{c}$ ).

Then, we detected PPAR $\gamma$ and C/EBP $\alpha$ at both the protein and mRNA levels after 14 days. Our research found that, compared with the control group, nocodazoletreated cells had increased levels of both PPAR $\gamma$ and C/ $\mathrm{EBP} \alpha$; however, cells treated with taxol showed the opposite results (Fig. 2d-h). These findings indicate that the microtubule network undergoes significant changes during adipogenesis and suggest that adipogenesis can may be affected by the presence or absence of microtubule fibers (Fig. 2i). More specifically, low-stress microtubules promoted adipogenesis, while high-stress microtubules had the opposite effect. Our results suggest that changes in the abundance of microtubule fibers may regulate adipogenesis in hASCs partly by affecting the expression of PPAR $\gamma$ and C/EBP $\alpha$.

\section{Actin stress fiber disruption during adipogenesis}

We examined the role of actin during adipogenesis of hASCs and found that continuous F-actin fibers gradually depolymerized to monomeric G-actin after the induction of adipocyte differentiation. Meanwhile, we observed changes in the cellular localization of F-actin from localization throughout the whole cell in undifferentiated controls to localization in the peripheral cytoplasm of differentiated cells. G-actin was at the center of cells near the nuclei (Additional file 4: Figure S3).

\section{How does the LINC complex change during hASC adipogenesis?}

Physical cues play an important role in the determination of lineage fate in stem cells $[4,19,20]$. Several studies have demonstrated that cytoskeletal organization governs nuclear deformation in response to mechanical stress, and that microtubules resist nuclear deformations in the major axis while actin resists deformations in the minor axis [21, 22]. To further elucidate the dynamic changes in the morphology around the nucleus as adipogenesis progresses in hASCs, we measured the minor/ major axis nuclear aspect ratio at selected time points (Fig. 3a). The closer that this ratio is to 1 , the more rounded the nuclei are (Fig. 3b, Additional file 3: Figure S4). The aspect ratio showed significant increase in the cells at day 4 and decrease at day 14 when compared with day $0(0.6767 \pm 0.01675 \mu \mathrm{m}, 0.7866$ $\pm 0.01713 \mu \mathrm{m}$, and $0.5778 \pm 0.02320 \mu \mathrm{m}$ at days 0,4 , and 14 , respectively; 30 cells for each time point, $P<0.001$ for day 4 and $P<0.001$ for day 14 compared with day 0 ) and showed significant changes in the cells at day 4 when compared with day 14 (30 cells for each time point, $P<0.001$ ). These findings indicate that during adipogenesis of hASCs the nuclei adapt to changes in the cytoskeleton by 
a

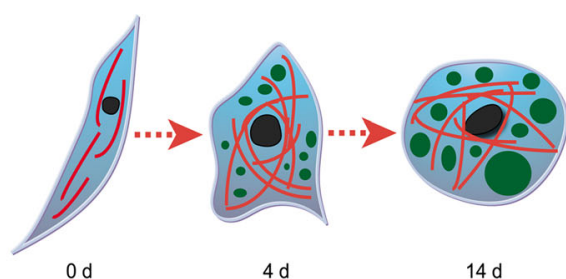

C Non-induced
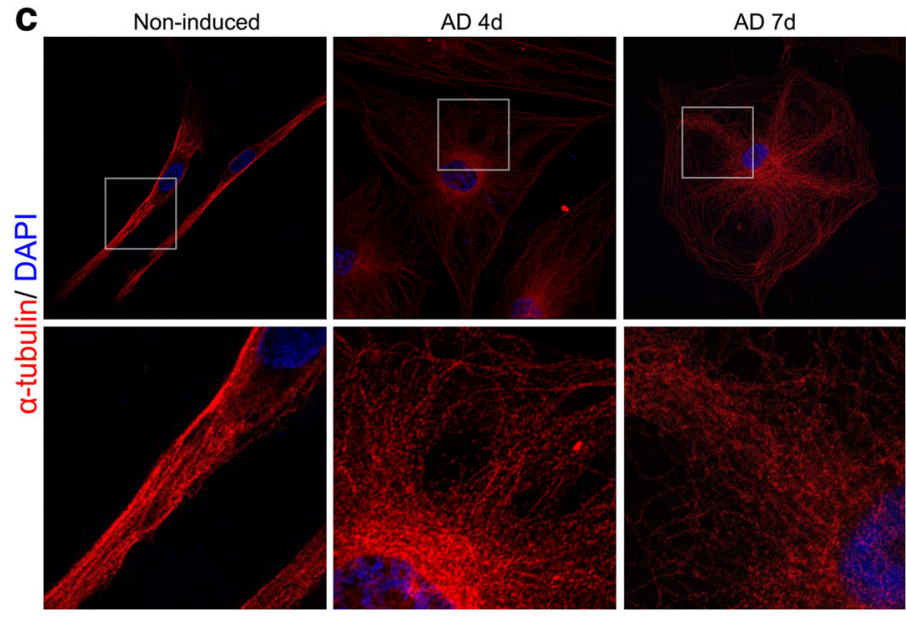

b cyossedeton
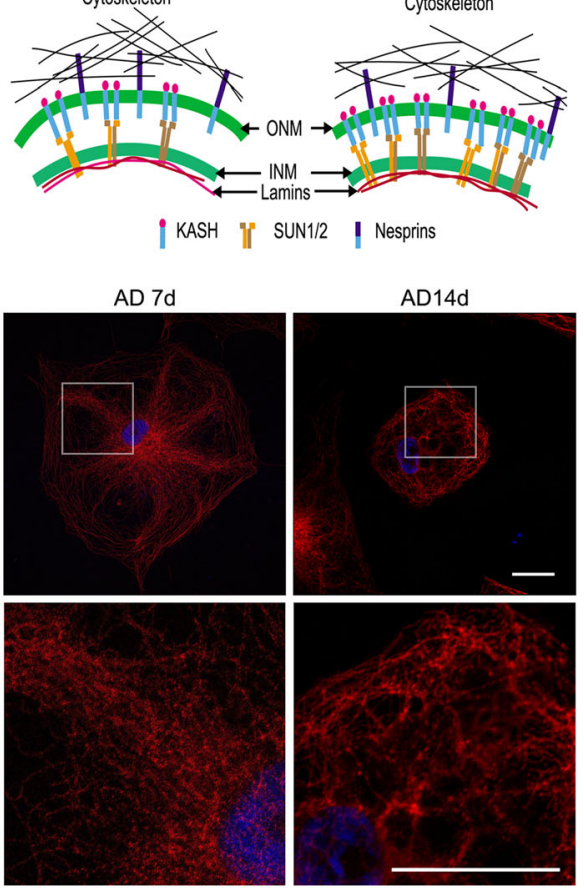

d

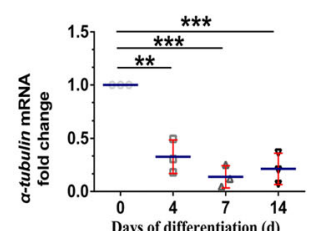

e

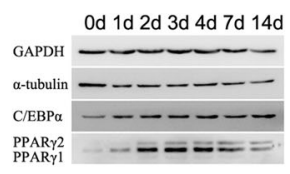

f

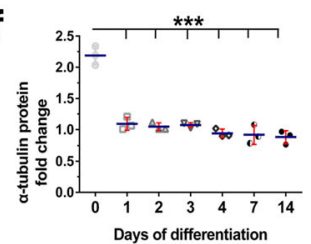

Fig. 1 Microtubule-based cytoskeleton remodeling during adipocyte differentiation (AD) and expression of microtubules (MTs) are induced during adipogenesis. a A schematic drawing depicting morphological changes in the MT network during adipocyte differentiation. Lipid droplets (LDs), MTs, and nuclei are indicated by green circles, red lines, and black circles, respectively. b Scheme of LINC complex remodeling during adipocyte differentiation. INM, inner nuclear membrane; ONM, outer nuclear membrane. c hASCs at days 0, 4, 7, and 14 after adipogenic cocktail treatment were fixed, costained with anti-a-tubulin (red) and for DNA (4',6-diamidino-2-phenylindole (DAPI); blue) and imaged using a confocal microscope for visualizing MTs and nuclei. Scale bars $=10 \mu \mathrm{m}$. $\mathbf{d}$ a-tubulin mRNA expression in hASCs at different days postinduction of adipogenesis was quantified by RT-qPCR; ${ }^{* *} P<0.01$, ${ }^{* *} P<0.001$, one-way ANOVA. e, f Whole-cell lysates of differentiation-induced hASCs were analyzed by Western blotting and probed with anti-a-tubulin antibody and for the adipogenic markers C/EBPa and PPARy. GAPDH was used to ensure equal loading. The graph shows average a-tubulin band intensities normalized to GAPDH; *** < 0.001, one-way ANOVA

regulating their morphology $[21,22]$. Furthermore, the shape of the nucleus seemed to be determined by the nucleoskeleton. In this study, we examined changes in Nesprin-1, Nesprin-3, SUN2, and lamin A in hASCs during adipogenesis.

\section{Nesprin-1 increased at the nuclear rim during early adipogenesis}

Nesprin-1 forms homodimers and specifically targets the nuclear envelope through a KASH domain [23]. Experimental evidence has shown that Nesprin-1 plays a vital role in the process of mesenchymal stem cell differentiation into cardiomyocyte-like cells [24]. Other studies have shown that Nesprin-1 strongly increases in early myogenesis during human muscle development [25]. However, it is not clear how Nesprin-1 changes during adipogenesis in hASCs. To determine how Nesprin-1 responds to adipogenesis, we assessed the Nesprin- 1 levels by RT-qPCR at various time points (days $0,4,7$, and 14) during adipogenic differentiation. The mRNA level of Nesprin-1 was increased in the early stage of adipocyte differentiation on day 4 (Fig. 4c).

Next, we performed immunofluorescence staining of hASCs at various stages of adipogenic differentiation to observe the morphologic changes and to evaluate the fluorescence intensity (Fig. 4a, b). Nesprin-1 localized along the nucleus to form a dot-like structure during adipogenesis. Nesprin-1 fluorescence intensity was 

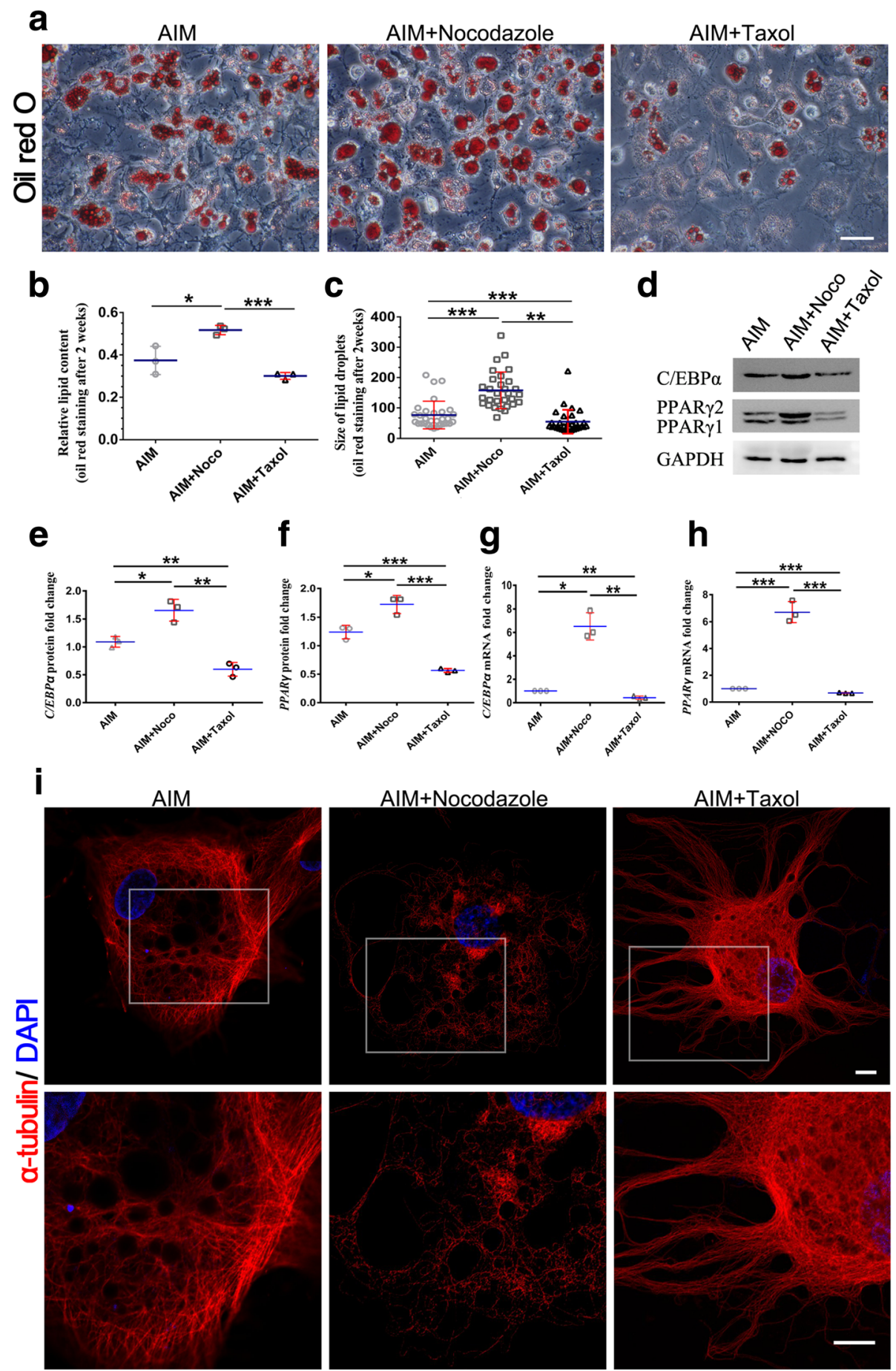

Fig. 2 Changes in microtubule mechanical status regulates adipogenesis. a Oil Red O staining for triglyceride content in hASCs after 14 days of differentiation treated with nocodazole $(0.1 \mathrm{\mu g} / \mathrm{ml})$, taxol $(10 \mathrm{nM})$, and adipogenic induction medium (AIM) only. Scale bar $=100 \mu \mathrm{m}$. b Intracellular triglyceride levels after 14 days of differentiation. Triglyceride content was normalized to total protein content and the control (AlM). One-way ANOVA was performed: ${ }^{*} P<0.05,{ }^{* *} P<0.001$. c Size of lipid droplets after 2 weeks of adipogenesis $(n>30$ cells per condition). $\mathbf{d}$ The levels of C/EBPa and PPARY were apparently increased in hASCs with nocodazole $(0.1 \mu \mathrm{g} / \mathrm{ml})$ (AlM+Noco) compared with the control group (AIM) at day 14 after adipogenic cocktail treatment. The taxol group (AIM+Taxol) showed the opposite results. e- $\mathbf{h}$ Western blotting and qPCR analysis of C/EBPa and PPARY in hASCs from the AIM+Noco, AIM+Taxol, and AIM groups after 14 days. i hASCs at 14 days after adipogenic cocktail treatment or with nocodazole $(0.1 \mathrm{\mu g} / \mathrm{ml})$ or taxol $(10 \mathrm{nM})$ were subjected to immunofluorescence staining of a-tubulin (red) and 4',6-diamidino-2-phenylindole (DAPI; blue). Scale bars $=10 \mu \mathrm{m}$ 

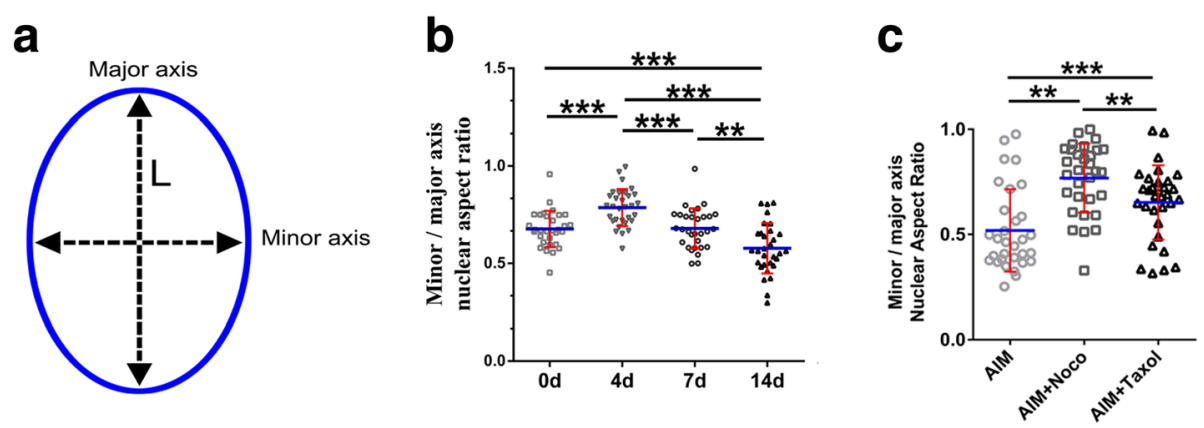

Fig. 3 Nuclear deformation during adipocyte development. a Process of nuclear deformation measurement. Nuclei stained with DAPI were observed for nuclear deformation. Software $\left(\right.$ Image $\left.^{6}\right)$ was then used to measure the length $(L)$ of the nuclear major or minor axis during adipogenic differentiation of hASCs at various time points. $\mathbf{b}$ The minor/major axis nuclear aspect ratio at selected time points, with more than 30 cells per time point; ${ }^{*} P<0.01,{ }^{* *} P<0.001$, one-way ANOVA. c The minor/major axis nuclear aspect ratio of the nocodazole (Noco) treatment group, taxol treatment group, and control group at day 14 , with more than 30 cells per group; ${ }^{* *} P<0.01,{ }^{* *} P<0.001$. AlM, adipogenic induction medium

enhanced when adipogenesis was initiated (day 4), and then decreased in intensity as the cell further matured (days 7 and 14), which is consistent with the RT-qPCR results. The aspect ratio showed significant enhancement in the cells at day 4 compared with days 0 and 14 .

\section{Nesprin-3 changed to a well-circumscribed circular huge ring-like structure during adipogenesis}

We also investigated whether Nesprin-3 changes during adipogenesis. First, we examined the morphological dynamics in Nesprin-3 during adipogenesis. Before adipogenesis, Nesprin-3 surrounded the nucleus in a loose structure, which changed to a well-circumscribed circular huge ring-like structure that not only surrounded the nucleus but also extended to the cell periphery, with an increased fluorescence intensity after exposure to inducers of adipogenic differentiation (Fig. 4d, e). Then, we analyzed Nesperin-3 expression at the mRNA level before and after the induction of adipogenic differentiation. Nesperin-3 mRNA (Fig. 4f) gradually increased during the first week and showed a greater than 12-fold increase after 14 days, consistent with the adipogenic markers. The protein level of Nesprin-3 was increased during adipogenesis (Fig. 4g, h). Taken together, our results revealed that during adipogenesis major morphological changes occurred in Nesprin-3, and staining density and protein expression were increased.

Lamin A/C scaffolding proteins inside the $\mathrm{NE}$ are mechanically coupled to the cytoplasmic cytoskeletal elements via LINC complex proteins. Previous studies have shown that lamin A plays a significant role in the differentiation of both osteoblasts and adipocytes [26, 27]. In our experiment, we observed that lamin A expression peaked when adipogenesis was initiated (day 4) (Additional file 5: Figure S5), similar to other LINC proteins. All these findings demonstrate that the nucleus undergoes rapid micromechanoenvironmental changes in the early differentiation phase of hASCs.

From previous results, we found that the expression levels of the Nesprin-1 and -3 genes increased during hASC adipogenesis, and both showed upregulated expression at day 4. In morphology, the Nesprin-3 structure changed from a loose structure around the nucleus to a huge ring-like structure that extended to the periphery starting at day 4 . These data indicate that Nesprin family members change their structure (especially Nesprin-3) and strength in response to adipogenesis. The initial stage of adipogenesis, at day 4 , is a crucial time window.

\section{Upregulation of SUN2 during adipogenesis}

To examine whether the cytoskeleton influences the nuclear response to hASC adipogenesis, we first analyzed SUN2 expression at the mRNA and protein levels during adipogenic differentiation of hASCs at various time points. As illustrated in Fig. 5a, SUN2 mRNA levels started to increase and showed significant values at day 4 and peaked at nearly sixfold by day 7 , followed by a small decline until day 14 after induction. During this period, we also examined changes in SUN2 protein expression during adipogenic differentiation. SUN2 exhibited an obvious increase (fourfold) at day 2 and then gradually increased, peaking at day 4 (sixfold). SUN2 protein levels returned to their original state after 2 weeks of induction (Fig. 5b, c). Before hASCs were transferred to adipogenic media, SUN2 not only localized to the nuclear rim but also appeared at the center of the nuclei. SUN2 began to localize to the edge of the nuclei and made its first appearance outside the nucleus after adipogenesis (Fig. 5e). 


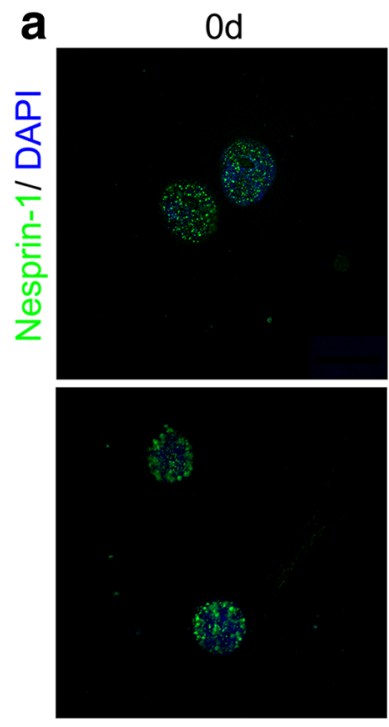

$7 d$

d

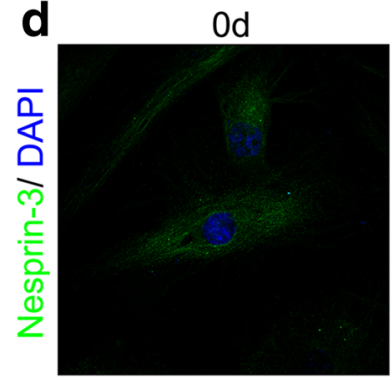

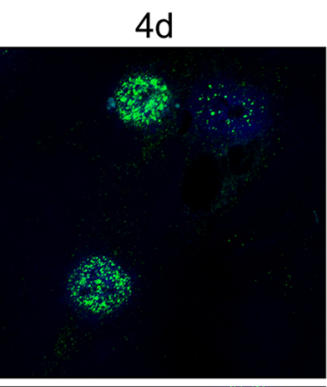

b
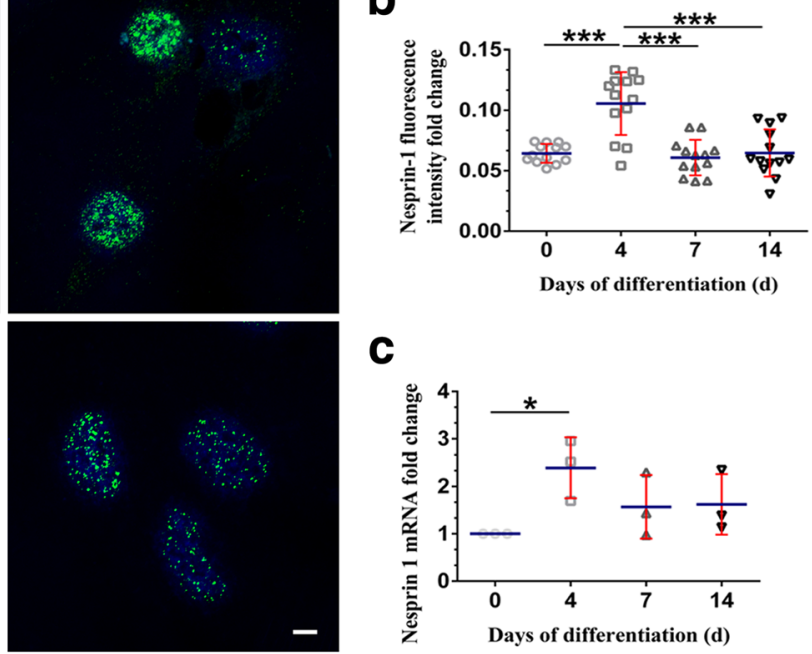

14d

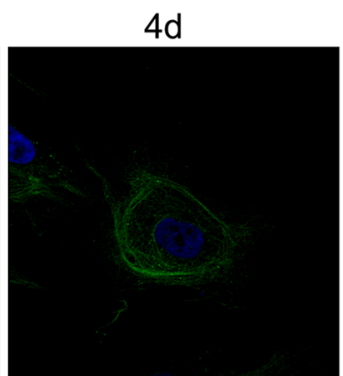

e

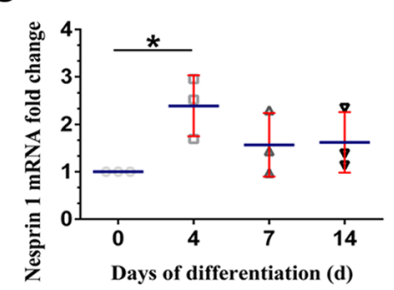

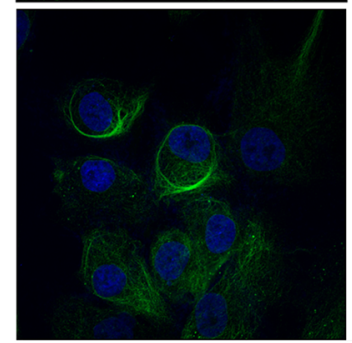

$7 d$

g

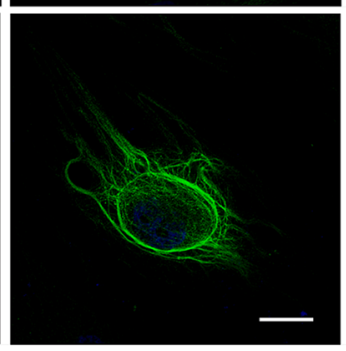

$14 d$

Nesprin-3

GAPDH

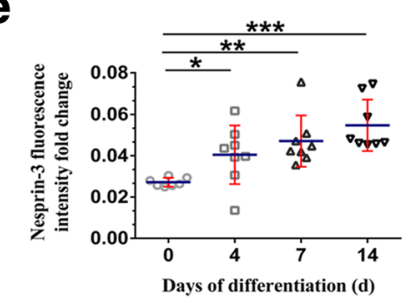

f

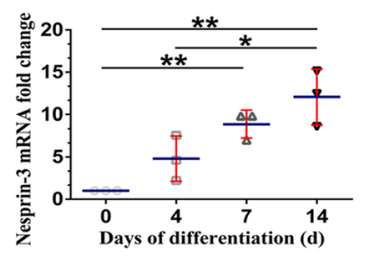

h

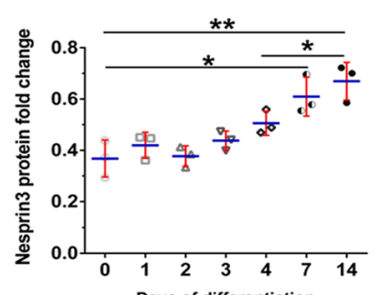

Fig. 4 LINC changes during adipocyte differentiation. a, b hASCs at selected time points were fixed, stained with an anti-Nesprin-1 (green) antibody and/or for DNA (4',6-diamidino-2-phenylindole (DAPI); blue), and imaged on a confocal microscope. Scale bars = $10 \mu \mathrm{m}$. The graph shows average Nesprin-1 (green) intensities derived from three independent differentiation experiments. $n>7$ cells; ${ }^{* * *} P<0.001$, one-way ANOVA. c Nesprin-1 mRNA expression in hASCs at different days postinduction of adipogenesis was quantified by RT-qPCR; $P>0.05$, one-way ANOVA. $\mathbf{d}$, e hASCs at selected time points were fixed, stained with the anti-Nesprin-3 antibody and imaged on a confocal microscope. Scale bars = $10 \mu \mathrm{m}$. The graph shows average Nesprin-3 (green) intensities derived from three independent differentiation experiments. $n>7$ cells; ${ }^{*} P<0.05$, ${ }^{* *} P<0.01$, ${ }^{* * *} P<0.001$, one-way ANOVA. $\mathbf{f}$ Nesprin-3 mRNA expression in hASCs at different days postinduction of adipogenesis was quantified by RT-qPCR; ${ }^{*} P<0.05$, ** $P<0.01$, oneway ANOVA. g, h Whole-cell lysates of differentiation-induced hASCs were submitted to Western blotting and probed with an anti-Nesprin-3 antibody. Anti-GAPDH was used to ensure equal loading. The graph shows the average Nesprin-3 band intensities normalized to glyceraldehyde-3-phosphate dehydrogenase (GAPDH) derived from three independent differentiation experiments; ${ }^{*} P<0.05,{ }^{* *} P<0.01$, ${ }^{* * *} P<0.001$, one-way ANOVA 
a

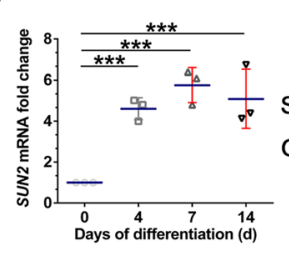

b

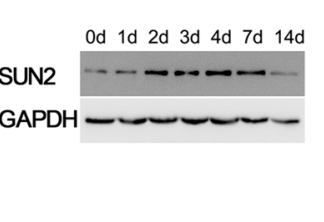

C

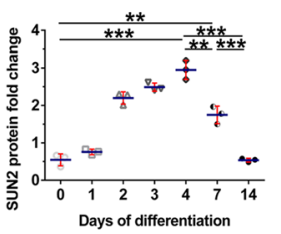

d

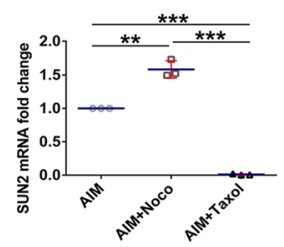

14d
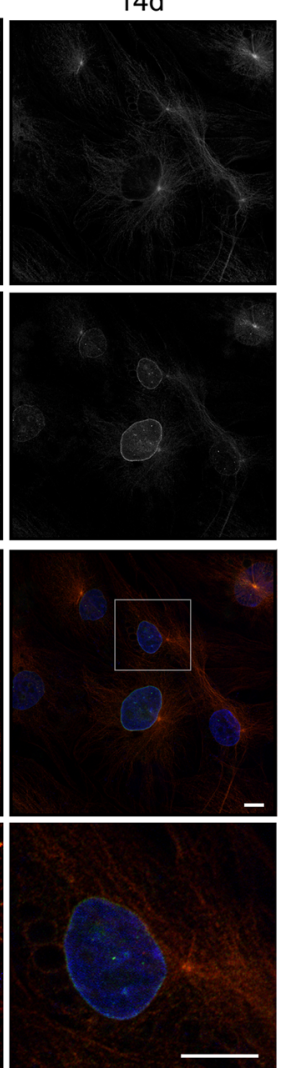

e

od

$4 d$

$7 d$
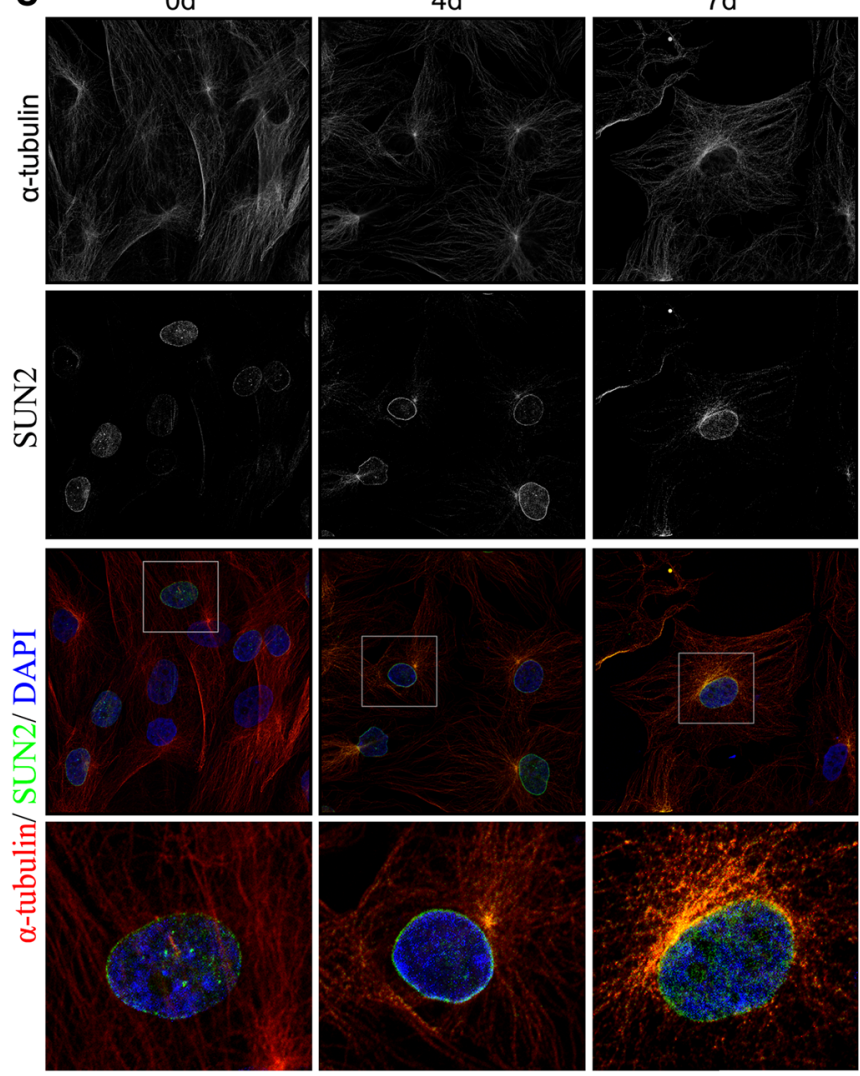

Fig. 5 Expression of SUN2 is increased during adipogenesis. a SUN2 mRNA expression in hASCs at different days postinduction of adipogenesis was quantified by RT-qPCR; **P $<0.01$, ***P $<0.001$, one-way ANOVA. b, c Whole-cell lysates of differentiation-induced hASCs were submitted to Western blotting and probed with anti-SUN2 antibody; ${ }^{* *} P<0.01$, ${ }^{* *} P<0.001$, one-way ANOVA. d SUN2 mRNA expression in hASCS with nocodazole (Noco; $0.1 \mathrm{\mu g} / \mathrm{ml})$ and taxol $(10 \mathrm{nM})$ after 4 days of adipogenesis was quantified by RT-qPCR; ${ }^{* *} P<0.01$, ${ }^{* *} P<0.001$, one-way ANOVA. e hASCs at selected time points were fixed, stained with the anti-SUN2 antibody, and imaged on a confocal microscope. Scale bars $=20 \mu m$. AlM, adipogenic induction medium; DAPI, 4',6-diamidino-2-phenylindole; GAPDH, glyceraldehyde-3-phosphate dehydrogenase

Previous research has shown that cytoplasmic microtubules were mechanically coupled to the nuclear heterochromatin through proteins embedded in the nuclear envelope and that the SUN-domain played a vital role in this process [28]. These findings led us to hypothesize that extranuclear SUN2 may be associated with microtubules, especially the microtubule-organizing center (MTOC). We costained SUN2 and $\alpha$-tubulin and found that they nearly overlapped. We then investigated the effect of microtubules on SUN2 gene expression (Fig. 5d) by treating cells with nocodazole and taxol after 4 days of adipogenesis. SUN2 expression increased with nocodazole $(P<0.01)$ while SUN2 expression decreased with taxol $(P<0.001)$ compared with that of day 4 hASCs cultured with only adipogenic media. We concluded that SUN2 undergoes significant changes during adipogenesis, indicating that microtubule-associated cytoskeletal changes may be involved in SUN2 regulation.

\section{SUN2 regulates adipogenic differentiation in hASCs by modulating PPAR $y$ and microtubules}

To determine whether SUN2 not only regulates but is also necessary for adipogenic differentiation, we transfected hASCs with a small interfering RNA (siRNA) pool 
against SUN2 (siSUN2) or a nontargeting control siRNA pool (siControl), and differentiation was induced $72 \mathrm{~h}$ post-transfection. Transfection with siSUN2 resulted in a 70\% knockdown of SUN2 mRNA over the entire period of differentiation (Fig. 6a). This knockdown resulted in the formation of fewer lipid droplets compared with that of the control, as shown by Oil Red O staining (Fig. 6c) and intracellular triglyceride content (Fig. 6b).

\section{Knockdown of sun2 changed the structure of perinuclear microtubules and induced microtubule upregulation during adipogenesis}

To determine whether there are some differences in the microtubule morphology and localization between the siSUN2 and siControl groups, we stained microtubules at an early stage (day 4 ) of adipocyte differentiation. On day 4 , the protein expression level of $\alpha$-tubulin in siSUN2-transfected cells was still greater than in the siControl treated cells (Fig. 6d, e). We observed changes in the cellular morphology of microtubules; microtubules appeared to be shorter, sparser, and had a vacuolar structure in the siControl group on day 4 (Fig. 7a, b, Additional file 6: Figure S6). In SUN2-depleted cells, microtubules maintained their integrity and rarely showed disruption. In contrast to the siControl group, in which the microtubules were around the nucleus, cells in the siSUN2 group had microtubules that formed a cap structure overlying the nuclei (Fig. 7a, b, Additional file 7: Figure S7). Our data showed that the size of siSUN2 cell maybe bigger than siControl cell although $P>0.05$ (Additional file 8: Figure S8).

We then analyzed $\alpha$-tubulin expression at the mRNA and protein levels during adipogenic differentiation of hASCs at day 4. At the cytoskeleton level, siSUN2 transfection resulted in a two- or threefold increase of $\alpha$ tubulin mRNA over the 4 days of differentiation (Fig. 7c). In addition, these results suggest that LINC complex disruption (knockdown of SUN2) can increase the expression of microtubules, which enhances the stiffness of hASCs and perturbs the perinuclear organization of microtubules ( $\alpha$-tubulin) in hASCs.

\section{Knockdown of sun2 induces PPARy downregulation during adipogenesis}

To investigate how SUN2 might contribute to adipogenesis, we performed Western blots and RT-qPCR analyses on day 4 of adipogenic differentiation in response to SUN2 knockdown. The mRNA levels of PPARy and C/ EBP $\alpha$ were assessed, with the expression of GAPDH at day 4 of adipogenic differentiation in the siControl group used to normalize the expression of the above genes. The relative expression of transcripts of the adipogenesis markers at various time points was further normalized to that of the siControl group at day 0 as control values to determine the relative fold-change in expression. PPAR $\gamma$ expression increased 50-fold in the siControl versus experimental cells at day 4 of adipogenic differentiation, while PPAR $\gamma$ expression in the siSUN2 group was at a very low level during the same period (Fig. 7c). C/EBP $\alpha$ levels were very similar with no significant differences between the siControl and siSUN2 cells (Fig. 7c). To evaluate the expression of adipogenesis markers and the cytoskeleton, we performed Western blot analyses at various stages (day 0, 4, 7) of adipogenic differentiation. Protein levels (Fig. 6d, e), as well as mRNA levels, of PPARy remained low in the siSUN2 group. $\mathrm{C} / \mathrm{EBP} \alpha$ expression gradually increased during the period of a week, with the siControl group level being higher than the siSUN2 group level. These results suggested that knockdown of SUN2 suppressed adipogenesis in hASCs, especially through the inhibition of PPAR $\gamma$ expression and the overexpression of $\alpha$-tubulin.

\section{Discussion}

In this article, we identified a previously unknown role for microtubules and the LINC complex component SUN2, and therefore the nuclear-cytoskeletal interface, in adipogenic differentiation of hASCs. Based on our data, we demonstrated that disrupting the physiological balance between the cytoskeleton and the LINC complex (especially SUN2) could impact the adipogenesis of hASCs in vitro. By disrupting microtubules and SUN2 in hASCs, we can modulate its adipogenic differentiation, indicating that biomechanical factors (include the cytoskeleton and LINC complex) participate in stem cell adipogenesis. Meanwhile, hASCs lacking sun 2 showed overexpression of microtubules and decreased PPAR $\gamma$ expression, thereby inhibiting the adipogenesis. Furthermore, knockdown of sun 2 changed the structure of perinuclear microtubules.

Cytoskeleton remodeling is an essential step for the morphological transition from fibroblastic preadipocytes to lipid-filled mature adipocytes during adipocyte development. It is also a gradually remodeling process that involves a feedback mechanism to adapt to the changing mechanical environment. The cytoskeleton is a dynamic structure that primarily consists of three types of proteins: microfilaments, microtubules, and intermediate filaments [29]. Previous research has shown that F-actin and intermediate filament vimentin remodeling occurs during stem cell adipogenesis [30]. Our data also suggested that microtubules are present around the nucleus at a high density and formed a cobweb-like structure. The cytoskeleton plays important roles in regulation of nuclear shape [21, 31], and microtubules resist nuclear deformations in the major axis while microfilaments resist deformation in the minor axis [22]. Recent work also identified the nucleus and its membrane as 
a

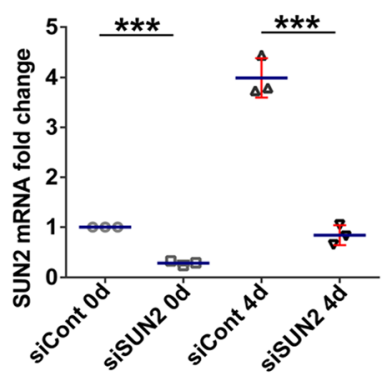

C
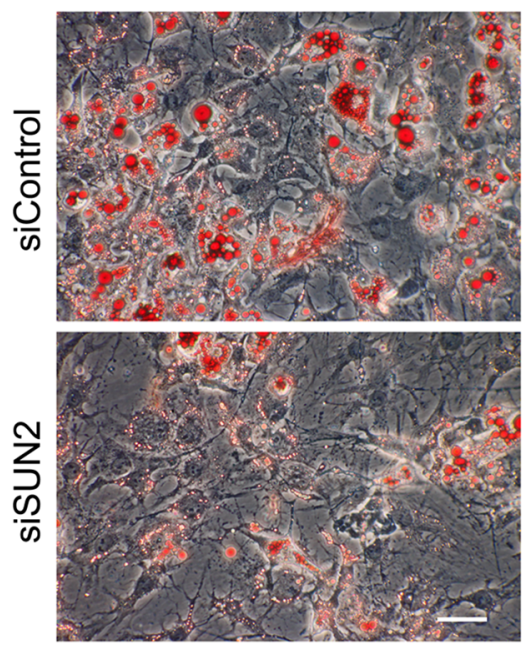

d b

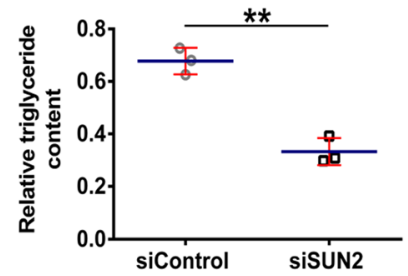

Oil red O/ GFP
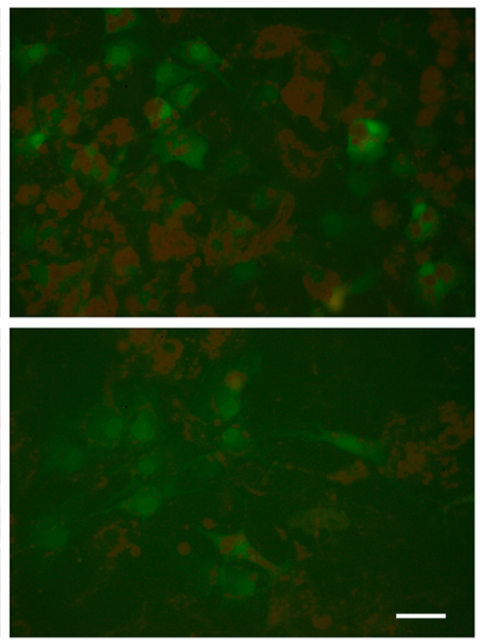

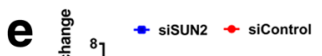

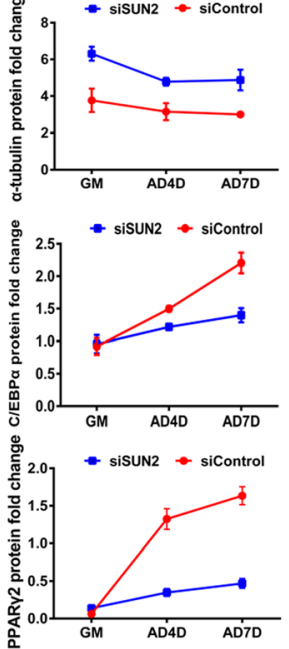

Fig. 6 Knockdown of SUN2 inhibits adipogenic differentiation (AD) of hASCs by downregulating adipogenesis-promoting signaling pathway PPARY and overexpressing microtubules (MTs). a RT-qPCR to determine knockdown efficiency in RNA samples; ${ }^{* * *} P<0.001$ ). b, c Oil Red O staining for triglyceride content in transfected hASCs after 7 days of differentiation. Scale bar $=100 \mu \mathrm{m}$. Intracellular triglyceride levels at day 7 of differentiation. Triglyceride content was normalized to total protein content and the control. Green fluorescent protein (GFP) represents transfected cells. An unpaired Student's $t$ test was performed; ${ }^{* *} P<0.01$. d, e hASC lysates of cells transfected with the indicated small interfering (si)RNAs were submitted to Western blotting and probed with an anti-SUN2 antibody and for the adipogenesis markers C/EBPa and PPARY. Glyceraldehyde-3-phosphate dehydrogenase (GAPDH) was used to ensure equal loading and normalized a-tubulin, C/EBPa, and PPARY2 protein levels. GM, growth medium 

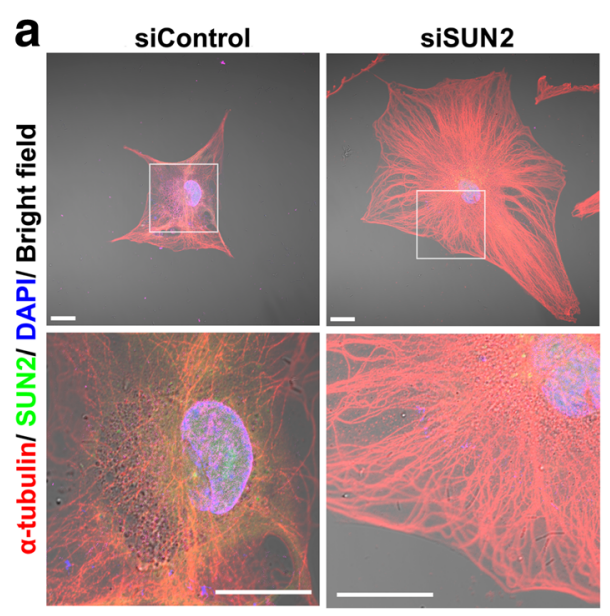

b
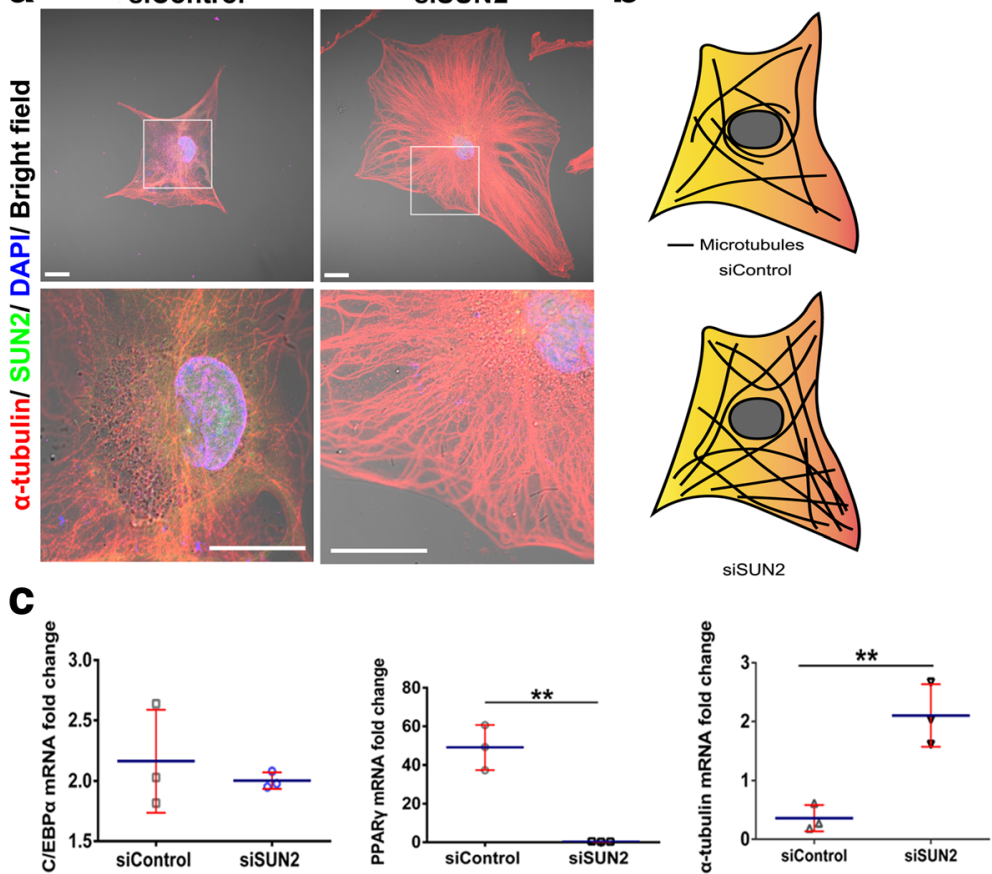

Fig. 7 LINC complex disruption perturbs the perinuclear organization of microtubules (MTs) in hASCs. a Immunofluorescence analysis of sun2knockdown hASCs and GFP controls. Cells were stained for a-tubulin (red), SUN2 (cherry), and DNA (4,6-diamidino-2-phenylindole (DAPI); blue). The boxed area shows the disturbed perinuclear MT network organization. Scale bar $=20 \mu \mathrm{m}$. b Model of the effect of sun2 loss on cytoskeletal organization in vitro. In siControl cells, MTs appeared to be shorter, sparser, and had a vacuolar structure. In siSUN2 cells, MTs maintained their integrity and rarely showed disruption. c RT-qPCR analysis of PPARy, C/EBPa, and a-tubulin. Tests were performed to compare small interfering (si)Control and siSUN2-treated samples at the same time points; ${ }^{* *} P<0.01$

mechanosensory organelles, where anchoring to the cytoskeleton via the LINC complex enables transmission of mechanical force between the nucleus and the cytoskeleton outward to the external microenvironment [6]. The nucleoskeleton, and associated tension inside and outside of the structure, alters the structure of the chromatin interfaces with the inner nuclear envelope and influences cell growth, division, and differentiation [32]. Cells often "soften" during adipogenesis, and hASCs need to rebalance their mechanical microenvironment to maintain the homeostasis, especially nuclear homeostasis. The importance of nuclear mechanics is observed during differentiation and development, when mechanical forces are necessary $[33,34]$.

LINC complex destruction can disturb the perinuclear organization of actin, vimentin, and keratin in various cell types [35-38]. Based on our data, the sudden increased density in LINC complexes (after 4 days of adipogenesis) suggests that the nucleus might reinforce itself to maintain its stability in response to mechanical environment changes, while the density of the cytoskeleton decreases. We hypothesized that the nucleoskeleton and the cytoskeleton might act as a mechanical network, similar to being on opposite sides of a seesaw, in which the strength of the nucleoskeleton falls while the strength of the cytoskeleton rises. A previous study showed that Lamin-A knockdown induced adipogenic differentiation, whereas overexpression enhanced osteogenic differentiation [27]. Interestingly, Lamin-A expression increased temporarily at the initiation of adipogenesis (day 4), although it declined in mature adipocytes (day 14) based on our data. Furthermore, our experiments suggest that SUN2 is particularly important for adipogenesis in hASCs and could not only alter microtubule perinuclear structure and enhance the microtubules but may also influence PPAR $\gamma$ transcription.

Microtubules have fundamental roles in many essential biological processes, such as intracellular transport which relies on the microtubule motor proteins kinesin and dynein $[39,40]$. In addition, LINC also played a direct role in mRNA export through the nuclear pore complex (NPC) in mammalian cells [41]. Microtubules interface with the LINC complex through the molecular motors dynein and kinesin. Nesprin-1 and Nesprin-2 isoforms interact with the microtubule motors kinesin-1 and dynein, although whether the binding is direct is unknown $[9,42]$. Mouse keratinocytes lacking SUN2 fail to correctly reorganize their microtubule cytoskeleton [43]. In conclusion, we hypothesized that microtubules and 
SUN2 both participate in intracellular transport of PPAR $\gamma$, and the MT-KASH-SUN2 axis might play an important role in PPAR $\gamma$ intracellular transport. Nevertheless, the interplay among the associated factors, including the mechanotransduction system, molecular mechanisms, and the fates of the hASCs during adipogenic differentiation, is extremely complex, and there is likely to be significant cross-talk between the various factors, all of which warrants further investigation.

Several studies demonstrated that the composition of the matrix used as a scaffold for stem cells may affect the fate of the cells; a stiffer substrate induces osteogenic differentiation, whereas a less stiff substrate leads to the adipogenic lineage fate $[4,44]$. These results may be mediated via changes in organization of the cytoskeleton and nuclear skeleton. Future studies are needed to demonstrate the organization of the cytoskeleton and nuclear skeleton in osteogenesis of ASCs. In future studies, we will observe the adipogenesis of ASCs under static mechanical stretching or cyclic tensile strain [45, 46] and on two-dimensional soft gel in a three-dimensional soft matrix.

\section{Conclusion}

Taken together, our results provide new insight into the relation between the dynamics of cell shape. Our results demonstrated that cross-talk is present between microtubules and SUN2, and this cross-talk plays a critical role in rebalancing the mechanical environment and is involved in the regulation of PPAR $\gamma$ transport during adipogenic differentiation of hASCs.

\section{Additional files}

Additional file 1: Figure S1. Identification of hASCs. (A) hASCs characterized for surface markers. (B) hASCs have the potential to differentiate towards osteocytes and adipocytes. Osteogenic differentiation: after 28 days of culture in the induction medium, hASCs stained positive for Alizarin red. Adipogenic differentiation: hASCs were subjected to adipogenic differentiation for 14 days. The formation of lipid droplets was visualized by Oil Red O staining (Scale bar $=200 \mu \mathrm{m}$ ). (TIFF $1470 \mathrm{~kb}$ )

Additional file 2: Figure S2. Time points of adipogeneic induced experiments. (TIFF $59 \mathrm{~kb}$ )

Additional file 3: Figure S3. Nuclear minor or major axis length changes. ( $A$ and $B$ ) Nuclear minor or major axis length changes at days 0 , 4,7 , and 14 after adipogenic cocktail treatment. The length of the nuclear major or minor axis during adipogenic differentiation of hASCs at various time points. $n>30$ cells; one-way ANOVA was performed: $* * * P<$ 0.001. (C and D) Nuclear minor or major axis length changes at days 0,4 , 7, and 14 after adipogenic cocktail treatment. The length of the nuclear major or minor axis after nocodazole or taxol treatment 14 days. $n>30$ cells; ${ }^{* *} P<0.01,{ }^{* * *} P<0.001$. (TIFF $190 \mathrm{~kb}$ )

Additional file 4: Figure S4. Actin stress fiber disruption during adipogenesis. hASCs at days $0,4,7$, and 14 after adipogenic cocktail treatment were fixed, costained with anti-F-actin (green) and DNA (blue) and imaged using a confocal microscope for visualizing MTs and nuclei (Scale bars $=20 \mu \mathrm{m})$. (TIFF $1550 \mathrm{~kb}$ )
Additional file 5: Figure S5. Lamin A changes during adipocyte differentiation. (A) hASCs at selected time points were fixed, stained with an anti-Lamin A (red) antibody and/or for DNA (blue), and imaged on a confocal microscope. Data points represent averages from three independent differentiation experiments. Scale bars $=10 \mu \mathrm{m}$. (B) The graph shows average Lamin A (red) intensities derived from three independent differentiation experiments. Error bars indicate SD. ${ }^{*} P<0.05,{ }^{* *} P<0.01$, ${ }^{* * *} P<0.001$, one-way ANOVA. (C) Whole-cell lysate of differentiationinduced hASCs were submitted to Western blotting and probed with an anti- Lamin A antibody. Anti-GAPDH was used to ensure equal loading. (D) The graph shows the average Nesprin-3 band intensities normalized to GAPDH derived from three independent differentiation experiments. Error bars indicate SD. (TIFF $678 \mathrm{~kb}$ )

Additional file 6: Figure S6. Immunofluorescence analysis of Lentivirusmediated transduction. For knockdown of human SUN2, we designed three different siRNAs for SUN2 from Genechem (Shanghai, China). It was proved that the sun 2 gene was knockdown in hASCs compared with the control group. (TIFF $3535 \mathrm{~kb}$ )

Additional file 7: Figure S7. LINC complex disruption perturbs the perinuclear organization of MTs in hASCs. Immunofluorescence analysis of sun2-knockdown hASCs and GFP control. Cells were stained for atubulin (red), SUN2 (cherry), and DNA (blue). The box area with disturbed perinuclear MT network organization (Scale bar $=20 \mu \mathrm{m}$ ). In siControl cells, MTs appeared to be shorter, sparser, and were changed into a vacuolar structure, while in siSUN2 cells, MTs maintained the integrity and rarely disrupted. (TIFF $1876 \mathrm{~kb}$ )

Additional file 8: Figure S8. The size of SUN2-knockdown (siSUN2) ASCs. Software (Image $J^{6}$ ) was used to measure the size of ASCs in the siSUN2 group and the siControl group. Error bars indicate SD. $P>0.05$, $n=5$ cells, one-way ANOVA. (TIFF $197 \mathrm{~kb}$ )

\section{Abbreviations}

AIM: Adipogenic induction medium; AMM: Adipogenic maintenance medium; ASC: Adipose-derived stem cell; C/EBPa: CCAAT/enhancer-binding protein a; DAPI: 4',6-Diamidino-2-phenylindole; DMEM: Dulbecco's modified Eagle's medium; FBS: Fetal bovine serum; GAPDH: Glyceraldehyde-3phosphate dehydrogenase; hASC: Human adipose-derived stem cell; KASH: Klarsicht, ANC-1, and Syne homology; KIF5B: Kinesin family member 5B; LD: Lipid droplet; LINC: Linker of the nucleoskeleton and cytoskeleton; MT: Microtubule; NE: Nuclear envelope; PBS: Phosphate-buffered saline; PPAR: Peroxisome proliferator-activated receptor; RT-qPCR: Real-time reverse transcription quantitative polymerase chain reaction; shRNA: Short hairpin RNA; SUN: Sad1, UNC-84; TBST: TBS with Tween 20

\section{Acknowledgements}

Not applicable.

\section{Funding}

This study was financially supported by the National Key R\&D Program of China (Jx Dai, No. 2017YFC1105000), and the Guangzhou major special projects of collaborative innovation of industry, university, and academic institute (201604040002 to JO).

\section{Availability of data and materials}

All the supporting data can be downloaded.

\section{Authors' contributions}

Conceived and designed the experiments: YtY, RQ, WH, JO, and JD. Performed the experiments: YtY, RQ, TF, XZ, YF, YCY, TD, and YP. Analyzed the data: $Y t Y, R Q$, and JD. Wrote the paper: $Y t Y, R Q, J O$, and JD. All authors read and approved the final manuscript.

\section{Ethics approval and consent to participate}

This study was approved by the Ethics Committee of the School of Basic Medical Sciences, Southern Medical University.

Consent for publication Not applicable. 


\section{Competing interests}

The authors declare that they have no competing interests.

\section{Publisher's Note}

Springer Nature remains neutral with regard to jurisdictional claims in published maps and institutional affiliations.

\section{Author details}

'Department of Anatomy, Guangdong Provincial Key Laboratory of Medicine and Biomechanics, School of Basic Medical Sciences, Southern Medical University, Guangzhou 510515, China. ${ }^{2}$ Departments of Obstetrics and Gynecology, Nanfang Hospital, Southern Medical University, Guangzhou 510515, China

Received: 13 December 2017 Revised: 26 February 2018 Accepted: 12 March 2018 Published online: 02 May 2018

\section{References}

1. Wu Y, Chen L, Scott PG, et al. Mesenchymal stem cells enhance wound healing through differentiation and angiogenesis. Stem Cells. 2007;25(10): 2648-59.

2. Frohlich M, Grayson WL, Marolt D, et al. Bone grafts engineered from human adipose-derived stem cells in perfusion bioreactor culture. Tissue Eng Part A. 2010;16(1):179-89.

3. Chen CS. Geometric control of cell life and death. Science. 1997;276(5317): 1425-8.

4. Engler AJ, Sen S, Sweeney HL, et al. Matrix elasticity directs stem cell lineage specification. Cell. 2006;126(4):677-89.

5. McBeath R, Pirone DM, Nelson CM, et al. Cell shape, cytoskeletal tension, and RhoA regulate stem cell lineage commitment. Dev Cell. 2004;6(4):483-95

6. Uzer G, Fuchs RK, Rubin J, et al. Concise review: plasma and nuclear membranes convey mechanical information to regulate mesenchymal stem cell lineage. Stem Cells. 2016;34(6):1455-63.

7. Dupin I, Camand E, Etienne-Manneville S. Classical cadherins control nucleus and centrosome position and cell polarity. J Cell Biol. 2009:185(5):779-86.

8. Sosa BA, Rothballer A, Kutay U, et al. LINC complexes form by binding of three KASH peptides to domain interfaces of trimeric SUN proteins. Cell. 2012;149(5):1035-47.

9. Zhang X, Lei $K$, Yuan $X$, et al. SUN1/2 and Syne/Nesprin-1/2 complexes connect centrosome to the nucleus during neurogenesis and neuronal migration in mice. Neuron. 2009;64(2):173-87.

10. Padmakumar VC, Libotte $T$, Lu W, et al. The inner nuclear membrane protein Sun 1 mediates the anchorage of Nesprin-2 to the nuclear envelope. Cell Sci. 2005:118(Pt 15):3419-30.

11. Thompson WR, Rubin CT, Rubin J. Mechanical regulation of signaling pathways in bone. Gene. 2012:503(2):179-93.

12. Almeida FV, Walko G, McMillan JR, et al. The cytolinker plectin regulates nuclear mechanotransduction in keratinocytes. J Cell Sci. 2015:128(24):4475-86.

13. Spiegelman BM, Farmer SR. Decreases in tubulin and actin gene expression prior to morphological differentiation of 3T3 adipocytes. Cell. 1982;29(1):53-60.

14. Nobusue $\mathrm{H}$, Onishi N, Shimizu T, et al. Regulation of MKL1 via actin cytoskeleton dynamics drives adipocyte differentiation. Nat Commun. 2014; 5:3368.

15. Franke WW, Hergt M, Grund C. Rearrangement of the vimentin cytoskeleton during adipose conversion: formation of an intermediate filament cage around lipid globules. Cell. 1987;49(1):131-41.

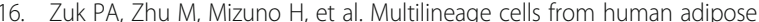
tissue: implications for cell-based therapies. Tissue Eng. 2001;7(2):211-28.

17. Serena C, Keiran N, Madeira A, et al. Crohn's disease disturbs the immune properties of human adipose-derived stem cells related to inflammasome activation. Stem Cell Rep. 2017;9(4):1109-23.

18. Shumay E, Gavi S, Wang HY, et al. Trafficking of beta2-adrenergic receptors: insulin and beta-agonists regulate internalization by distinct cytoskeletal pathways. J Cell Sci. 2004;117(Pt 4):593-600.

19. Yourek G, McCormick SM, Mao JJ, et al. Shear stress induces osteogenic differentiation of human mesenchymal stem cells. Regen Med. 2010;5(5):713-24.

20. Arnsdorf EJ, Tummala P, Kwon RY, et al. Mechanically induced osteogenic differentiation - the role of RhoA, ROCKII and cytoskeletal dynamics. J Cell Sci. 2009;122(Pt 4):546-53.
21. Tremblay D, Andrzejewski L, Leclerc A, et al. Actin and microtubules play distinct roles in governing the anisotropic deformation of cell nuclei in response to substrate strain. Cytoskeleton. 2013;70(12):837-48.

22. Haase $\mathrm{K}$, Macadangdang $\mathrm{JKL}$, Edrington $\mathrm{CH}$, et al. Extracellular forces cause the nucleus to deform in a highly controlled anisotropic manner. Sci Rep. 2016;6(1):21300

23. Zhang Q, Skepper JN, Yang F, et al. Nesprins: A novel family of spectrinrepeat-containing proteins that localize to the nuclear membrane in multiple tissues. J Cell Sci. 2001;114(Pt 24):4485.

24. Yang $W$, Zheng $H$, Wang $Y$, et al. Nesprin-1 has key roles in the process of mesenchymal stem cell differentiation into cardiomyocyte-like cells in vivo and in vitro. Mol Med Rep. 2015;11(1):133

25. Randles KN, Lam LT, Sewry CA, et al. Nesprins, but not sun proteins, switch isoforms at the nuclear envelope during muscle development. Dev Dynam. 2010;239(3):998-1009.

26. Bermeo S, Vidal C, Zhou H, et al. Lamin A/C acts as an essential factor in mesenchymal stem cell differentiation through the regulation of the dynamics of the Wnt/ß-Catenin pathway. J Cell Biochem. 2015;116(10):2344-53.

27. Swift J, Ivanovska IL, Buxboim A, et al. Nuclear lamin-A scales with tissue stiffness and enhances matrix-directed differentiation. Science. 2013; 341(6149):1240104.

28. King MC, Drivas TG, Blobel G. A network of nuclear envelope membrane proteins linking centromeres to microtubules. Cell. 2008;134(3):427-38.

29. Burridge K, Wittchen ES. The tension mounts: stress fibers as forcegenerating mechanotransducers. J Cell Biol. 2013;200(1):9-19.

30. Kanzaki M, Pessin JE. Caveolin-associated filamentous actin (Cav-actin) defines a novel f-actin structure in adipocytes. J Biol Chem. 2002;277(29):25867-9.

31. Guolla $L$, Bertrand $M$, Haase $K$, et al. Force transduction and strain dynamics in actin stress fibres in response to nanonewton forces. J Cell Sci. 2012; 125(Pt 3):603-13.

32. Ungricht R, Kutay U. Mechanisms and functions of nuclear envelope remodelling. Nat Rev Mol Cell Biol. 2017;18(4):229-45.

33. Rowat AC, Lammerding J, Ipsen JH. Mechanical properties of the cell nucleus and the effect of emerin deficiency. Biophys J. 2006;91(12): 4649-64.

34. Brandt A, Papagiannouli F, Wagner N, et al. Developmental control of nuclear size and shape by kugelkern and kurzkern. Curr Biol. 2006;16(6): 543-52.

35. Postel R, Ketema M, Kuikman I, et al. Nesprin-3 augments peripheral nuclear localization of intermediate filaments in zebrafish. J Cell Sci. 2011;124(Pt 5):755-64

36. Chambliss AB, Khatau SB, Erdenberger N, et al. The LINC-anchored actin cap connects the extracellular milieu to the nucleus for ultrafast mechanotransduction. Sci Rep. 2013;3(1):1087

37. Lombardi ML, Lammerding J. Keeping the LINC: the importance of nucleocytoskeletal coupling in intracellular force transmission and cellular function. Biochem Soc Tran. 2011:39(6):1729-34.

38. Rashmi RN, Eckes B, Glockner G, et al. The nuclear envelope protein Nesprin-2 has roles in cell proliferation and differentiation during wound healing. Nucleus-Phila. 2012;3(2):172-86.

39. Caviston JP, Holzbaur ELF. Microtubule motors at the intersection of trafficking and transport. Trends Cell Biol. 2006;16(10):530-7.

40. Akhmanova A, Steinmetz MO. Control of microtubule organization and dynamics: two ends in the limelight. Nat Rev Mol Cell Biol. 2015;16(12):711-26.

41. Antonin W, Ellenberg J, Dultz E. Nuclear pore complex assembly through the cell cycle: regulation and membrane organization. FEBS Lett. 2008; 582(14):2004-16.

42. Yu J, Lei $K$, Zhou M, et al. KASH protein Syne-2/Nesprin-2 and SUN proteins SUN1/2 mediate nuclear migration during mammalian retinal development. Hum Mol Genet. 2011:20(6):1061-73.

43. Stewart RM, Zubek AE, Rosowski KA, et al. Nuclear-cytoskeletal linkages facilitate cross talk between the nucleus and intercellular adhesions. J Cell Biol. 2015;209(3):403-18

44. Rowlands AS, George PA, Cooper-White JJ. Directing osteogenic and myogenic differentiation of MSCs: interplay of stiffness and adhesive ligand presentation. Am J Physiol Cell Physiol. 2008;295(4):C1037-44.

45. Wang J, Wang CD, Zhang $N$, et al. Mechanical stimulation orchestrates the osteogenic differentiation of human bone marrow stromal cells by regulating HDAC1. Cell Death Dis. 2016;7:e2221.

46. Shoham N, Gottlieb R, Sharabani-Yosef O, et al. Static mechanical stretching accelerates lipid production in 3T3-L1 adipocytes by activating the MEK signaling pathway. Am J Physiol Cell Physiol. 2012;302(2):C429-41. 